\title{
Modelling of new vehicle suspension concept with integrated electric drive
}

\author{
Jakub Tobolár $\check{r}^{a} \quad$ Jakob Müller $^{b} \quad$ Alfred Pruckner ${ }^{b}$ \\ ${ }^{a}$ German Aerospace Center (DLR), Institute of Robotics and Mechatronics, Germany; \\ ${ }^{b}$ BMW Group Forschung und Technik, Munich, Germany
}

\begin{abstract}
In the last decade an electrification of the powertrain became the significant trend in the passenger cars' development. Beside hybrid electric powertrains there is also a variety of solutions for pure electric cars. The presented paper introduces a Modelica model of an electric vehicle solution with rear driven wheels. The suspension model containing an individual electric drive placed close to the wheel will be discussed with focus on different modelling aspects. Moreover, some typical characteristics of suspension will be presented.

Keywords: Modelica; vehicle suspension; planetary gerbox; electric car; connecting multibody with onedimensional
\end{abstract}

\section{Introduction}

In the last years, the electrification of the powertrain of passenger cars became one of the huge challenges for the vehicle developers. This is the consequence of the legislative demand to reduce the emissions and of customer wish to reduce fuel consumption as well.

Several solutions for the hybrid electric vehicles exist such as parallel or serial arrangement of internal combustion engine and electric drive. Whereas such solutions are preferred for mass-production vehicles the pure electric vehicles are still designed in significantly lower series.

Electric cars commonly utilise either vehicle body mounted motors or in-wheel drives. For the first one the gearbox and drive shafts transmit the drive torque to the wheels. An alternative concept for electric car suspension with drive close to the wheel was developed in the joint research project of BMW Group Forschung und Technik, DLR and Schaeffler Group. This concept should utilise advantages of abovementioned common solutions and additionally minimise the required space needed for all components including battery.

To investigate the behaviour of the suspension in the early design stage the mutlibody model was created. Later, a model of electric motor was additionally utilized in an overall vehicle model. Based on the vehicle model a drive control strategy was developed and optimized for various drive manoeuvers.

The presented paper focuses on different aspects of the modelling in the early design stage.

\section{Suspension concept and design}

Typical for BMW vehicles, the developed driven suspension was designed for the rear axle, see the resulting overall design as shown in Figure 1. The basic idea was to couple the electric drive fixed on vehicle body with suspended wheel by means of a gearbox integrated in the wheel, see [1].

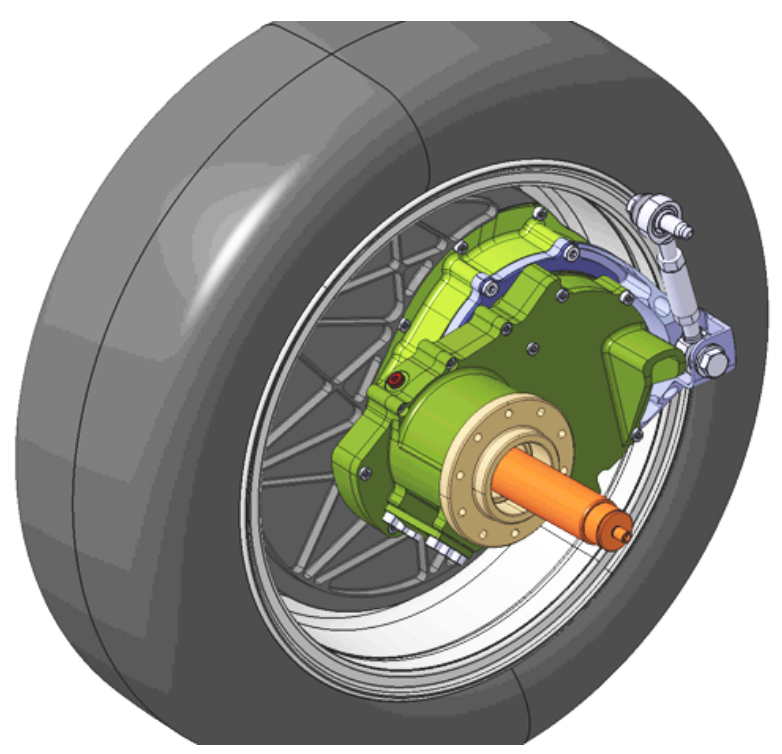

Figure 1: Overall view of the presented rear suspension (electric drive not displayed) 
For the wheel guidance there is used a mechanism which can be simply imagined as double pendulum, cf. Figure 2. A swing arm rotating about the axis $n_{1}$ oriented in lateral direction is fixed on the vehicle body. The wheel carrier is joined rotationally to this swing arm, whereby the axis $n_{2}$ of rotation points to the lateral direction as well. To constrain one redundant degree of freedom, the wheel carrier is additionally linked to the vehicle body. The link is placed before the rotational axis $n_{2}$ of the carrier. To tune the kinematic characteristics of the suspension the orientation of the two rotational axes $n_{1}$ and $n_{2}$ and the position of the link mounting points can be changed.

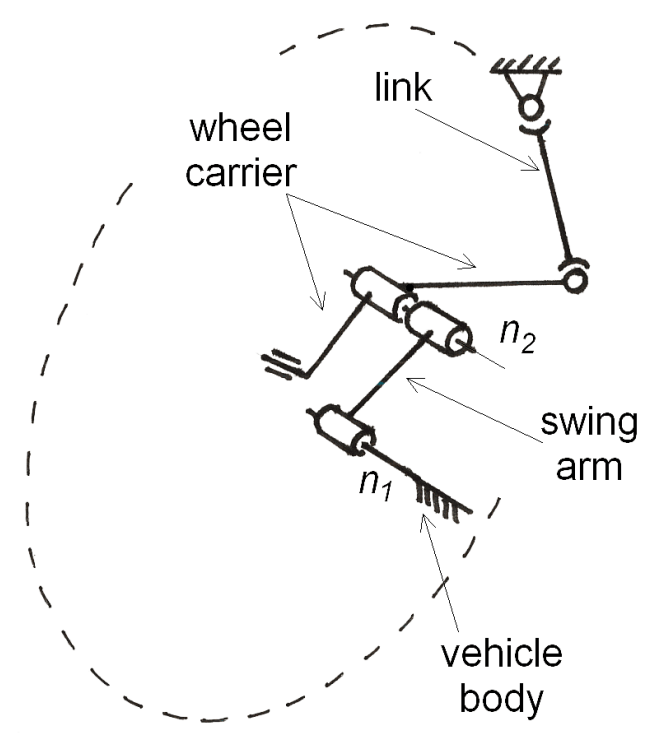

Figure 2: Structure of the wheel guidance mechanism

As mentioned above, the in-wheel gear was suggested for the power transmission to minimise the required space in a vehicle. Due to this solution the electric drive can be placed close to the wheel at the rotational axis $n_{1}$ of the swing arm. The driving torque is transmitted from the drive pinion to the lay shaft on axis $n_{2}$ and then to the wheel rim. To reach the desired ratio from drive pinion to wheel, there are two "planet" gearwheels on the lay shaft, one in contact with the pinion and the other in contact with the ring wheel.

To minimise required space a rotational damper and spiral spring were employed in suspension. Using such rotational elements was the best way to exploit the large rotational movements of suspension during deflection and rebound. The spring was placed on axis $n_{1}$ and designed to react the torques acting on the swing arm. It is supported directly on the vehicle body.
The damper is connected to the swing arm and wheel carrier instead. Placed on the same axis $n_{2}$ like the lay shaft, the rotational velocity of the damper is approximately double of that of the spring.

Especially for acoustic reasons, there are used elastic bushings and a subframe, too, as usual in the vehicle design. Each of the suspensions is coupled by means of bushings on the subframe thus constituting one axle unit. Finaly, the complete subframe is mounted elastically on the vehicle body.

\section{Modelling}

The aim of the modelling was to create the multibody model of the suspension and of the complete vehicle to perform common analyses during the early design stage. Additionally, the multibody model was used to tune the vehicle dynamics control.

In order to promote easy interoperability with the various automotive libraries not only from DLR, the created Modelica library was consequently based on the VehicleInterfaces standards, see [2]. The VehicleInterfaces focuses on standardising the assemblies interface definitions without enforcing a standard vehicle model architecture, so that the same assembly models can be reused in different model architectures. For example, the chassis assembly uses the same interface definition regardless of it being a basic onedimensional (1D) longitudinal model or a complex multibody vehicle dynamics model.

All the assembly models were created based on the idea of template and parametrised models as also utilised in the PowerTrain library from DLR, see [3], [4] and [5]. Therefore, every Modelica subpackage with assemblies such as suspensions or steerings contains template models - i. e. assembly models of different level of detail and for diverse purposes. Various meaningfully parametrised models of realistic assemblies are then inherited from such template models and used in an overall vehicle architecture thus representing a particular vehicle model.

In the following the modelling of the discussed suspension will be described in more detail.

\subsection{Wheel guidance}

The wheel guidance mechanism was realised as a multibody model with two rigid bodies and two rotational joints, each one for the swing arm and for the wheel carrier. The movement of the wheel carrier was constrained with the link modelled as the Universal- 
Spherical joint from Multibody package of Modelica standard library. The final design of the suspension utilizes slightly skew rotational axes to achieve suitable kinematics common for rear axles of passenger cars, see camber and toe angle characteristics in Figure 3.
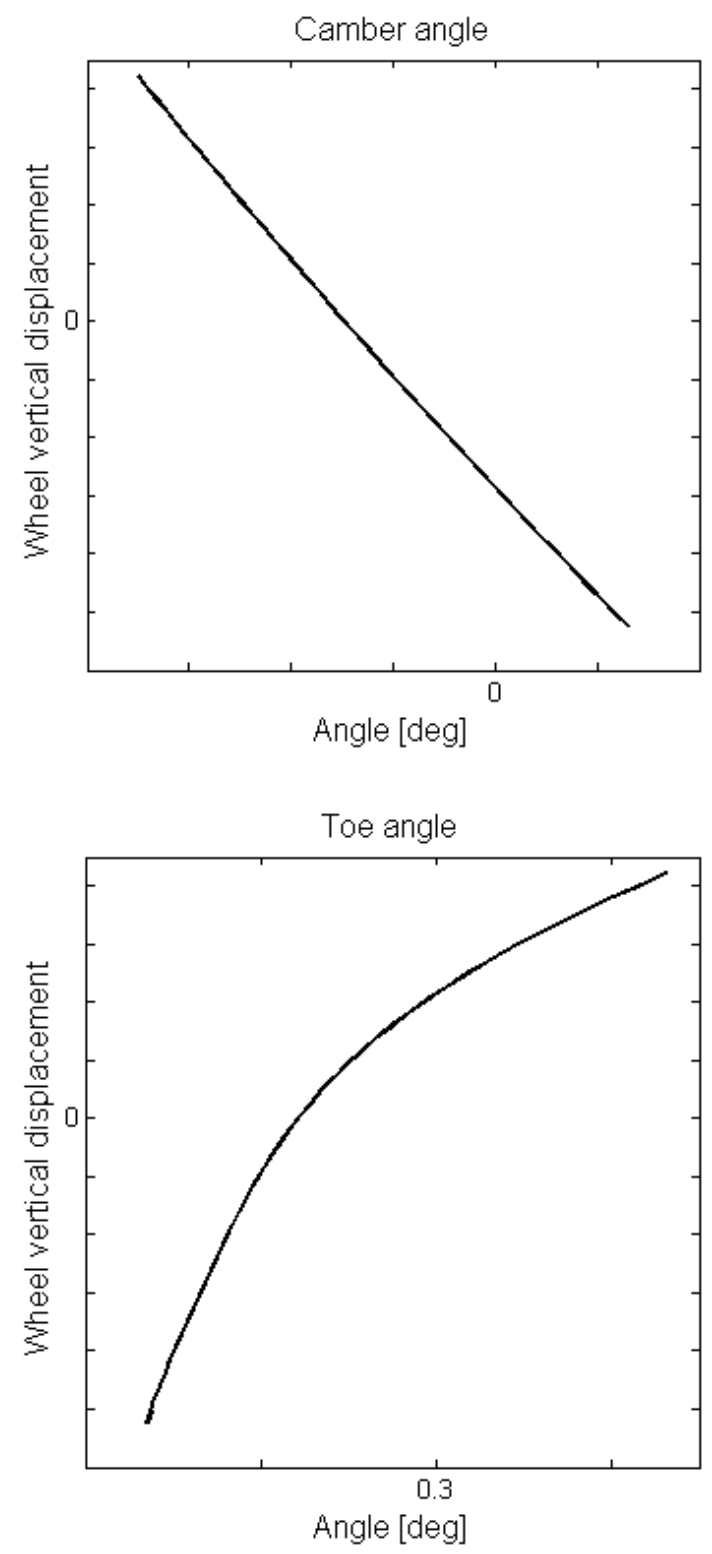

Figure 3: Camber and toe angle of suspension

\subsection{Gearbox}

The two-stage gearbox was modelled by means of two one-dimensional rotational PlanetPlanet models (see [5]) from PowerTrain library, cf. blocks sunPlanet and planetRing in Figure 4. The sunPlanet represents the first stage from drive pinion to the lay shaft, the planetRing is used for the second stage from lay shaft to the ring gearwheel which is fixed on the wheel rim. This configuration is similar to that of a planetary gearbox.

In the multibody model of the suspension the rotational joints of the wheel as well as of the wheel carrier connect the respective body to the predecessory one. It means, that the joint angles reflect relative rotation of wheel to wheel carrier and of wheel carrier to swing arm, respectively. On the contrary, the angles of sun and planet gearwheels and of carrier in the PlanetPlanet model are absolute angles as adequate for a 1D rotational mechanics. Therefore, a 1D sub-model was added which calculates necessary relative angles to connect the 1D gearbox and multibody suspension in a correct way. This sub-model was called RotationalAdd and used two times in the gearbox model, see the blocks called rotAdd2_i, $i=1,2$ in Figure 4.

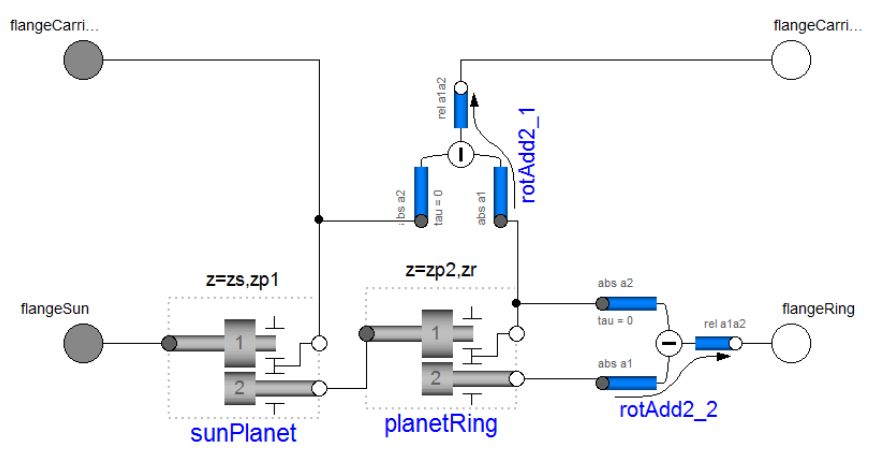

Figure 4: One-dimensional Modelica model of the gearbox

This sub-model for rotational additon has three flanges: 1, 2 and 12. The corresponding flange angles $\phi_{1}, \phi_{2}$ and $\phi_{12}$ result from simple kinematics:

$$
\phi_{2}=\phi_{1}+\phi_{12}
$$

The appropriate flange torques yield

$$
\begin{aligned}
\tau_{1} & =0 \\
\tau_{2} \omega_{2}+\tau_{12} \omega_{12} & =0
\end{aligned}
$$

The equations (1) and (2) both summarise power balance on flanges 1,2 and 12. Herewith, the power flow between 2 and 12 must be in balance. At the flange 1, in contrast, no power flow may be realised.

Let us focus now on the connection of the sunPlanet component as first stage of the gearbox to the swing arm joint. The swing arm body is identical with the carrier of sunPlanet. For swing arm joint the revolute joint from Modelica standard library called Modelica.Mechanics.MultiBody.Joints.Revolute could 
be used, cf. [6]. This revolute joint has two multibody ( $a$ and $b$ ) and two 1D rotational (axis and support) connectors. Simplified described, the following torque balance is adopted between the connectors:

$$
\begin{aligned}
\tau_{M B S, a} & =-\mathbf{T}_{a b} \tau_{M B S, b}, \\
\tau_{1 \mathrm{D}, a x i s} & =-\tau_{M B S, b} \mathbf{n}_{a x i s},
\end{aligned}
$$

with transformation matrix $\mathbf{T}_{a b}$ from multibody frame $b$ to frame $a$ and vectors of cut torques $\tau_{M B S, a}$ and $\tau_{M B S, b}$ in frames $a$ and $b$, respectively, and normalised vector $\mathbf{n}_{\text {axis }}$ of rotation axis. It should be noted that the torque from 1D flange support does not apply within this joint. Consequently, when connecting 1D flange axis to flangeCarrier from 1D gearbox (see connectors in Figure 4) the scalar carrier torque $\tau_{\text {flangeCarrier }}=\tau_{1 \mathrm{D} \text {,axis }}$ would be applied on multibody frame $b$ and supported on frame $a$. However, this is unrealistic since for idealised frictionless joints the supporting torque can only be realised by the torque source, i. e. by the electric drive.

Therefore, the rotational joint equations are modified as follows:

$$
\begin{gathered}
\tau_{M B S, a}+\tau_{1 \mathrm{D}, \text { support }} \mathbf{n}_{\text {axis }}=-\mathbf{T}_{a b}\left(\tau_{M B S, b}+\tau_{1 \mathrm{D}, \text { axis }} \mathbf{n}_{\text {axis }}\right), \\
\tau_{1 \mathrm{D}, \text { axis }}=-\tau_{M B S, b} \mathbf{n}_{\text {axis }} .
\end{gathered}
$$

With such a definition, the torque from 1D flange axis only applies on multibody frame $b$ and the torque from 1D support on frame $a$. Consequently, the carrier torque from 1D gearbox only applies on the swing arm and not on the predecessory body. The connection of the second stage of the gearbox to the wheel carrier joint is arranged in the same way.

The final connection of the total gearbox model with the multibody components is depicted in Figure 5.

\subsection{Spiral spring and damper}

For suspension the rotational spiral spring was connected between vehicle body and swing arm. It was designed to optimally support reaction forces and torques acting in the swing arm mounting. Especially, the torque about the swing arm rotational axis and the vertical force were considered, both resulting from tyre/road contact. The rotational stiffnes of the realised spring is nearly constant over the whole wheel deflection range.

Both cut torques and cut forces on the spring mounts are dependent on their relative deflection, i. e. their relative orientation and displacement. These dependencies were modelled by means of multi-dimensional tables within one multibody force element. The tables

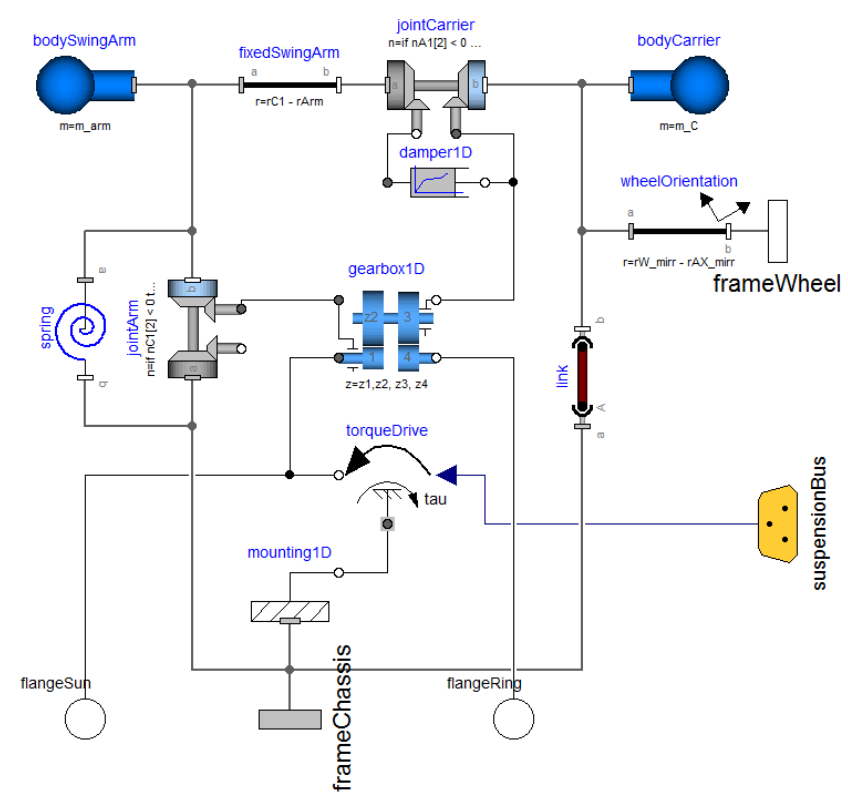

Figure 5: Modelica model of suspension (some marginal elements are not shown)

were generated previously on the base of a finite element spring model.

The rotational damper acting between swing arm and wheel carrier was modelled as one-dimensional non-linear damper.

\subsection{Axle subframe and bushings}

On the rear axle, each of the suspensions is mounted on a module carrier. Each of these module carriers is then elastically mounted on a subframe which again is elastically mounted on the vehicle body.

The elastic bushings in the mounts are modelled as force elements. Generally, it is formulated in such a way that the forces and torques depend linearly on the relative position and orientation angles of its connectors, respectively, and on their derivatives. The bushings operate at small angles, i. e. at angles with a magnitude less then $5^{\circ}$. This fact was considered to simplify the calculations.

\section{Suspension kinematics and brake support angle}

In our project, the functionality of the suspension was proven in various tests, both virtual and real. Within this section the suspension kinematic characteristics will be discussed in more detail. 
As already shown in Figure 3, the progress of camber and toe angles was tuned when modifying the orientation of rotational axes $n_{1}$ and $n_{2}$ of the swing arm and the wheel carrier, respectively.

Let us now exploit next typical characteristics - the support angle $\varepsilon_{B}$ at braking. Together with the brake support angle of front suspension and the height of centre of gravity of the vehicle it is crucially responsible for the amount of vehicle pitch movement during braking. According to [7], this angle can be calculated by means of the translational velocity $\mathbf{v}_{W}^{*}$ at the "virtual" tire/road contact point as

$$
\tan \varepsilon_{B}= \pm \frac{v_{W x}^{*}}{v_{W z}^{*}}
$$

Such virtual contact point is considered on the wheel with blocking brake during wheel deflection and rebound. In a case of conventional vehicle with brakes mounted on the wheel carrier this means that the wheel can be virtually fixed on the carrier during computer aided investigation of $\varepsilon_{B}$. For practical reasons the point can simply be considered to be on the wheel carrier, too. On the contrary, when the brake is mounted otherwise, e. g. on the vehicle body, such virtual blocking must be regarded in a correct way.

This is also the case for the described suspension. Since the suspension is considered to have no conventional friction brake, the brake torque will only be realised via electric drive. Therefore, in the simulation the drive pinion was fixed for the brake support angle analysis. In Figure 6 the trajectory of the virtual contact point is depicted for our case compared to the point trajectory at conventionally braked wheel.

The analysis proved that the brake support angle $\varepsilon_{B}$ depends not only on the suspension geometry but additionally on the gear ratio $i_{\text {Total }}$ as depicted by means of three curves in Figure 7 for increasing ratio.

\section{Conclusions}

The paper gives an overview of the new vehicle suspension concept with integrated gearbox and electric drive and focuses especially on modelling aspects. In the model, the multibody suspension parts are combined with one-dimensional rotational elements for two-stage gearbox thus enabling efficient simulation. For proper interaction between such one-dimensional and multibody parts new Modelica models were introduced.

Besides the wheel guidance functionality, the particular models of gearbox, spiral spring and bushings are

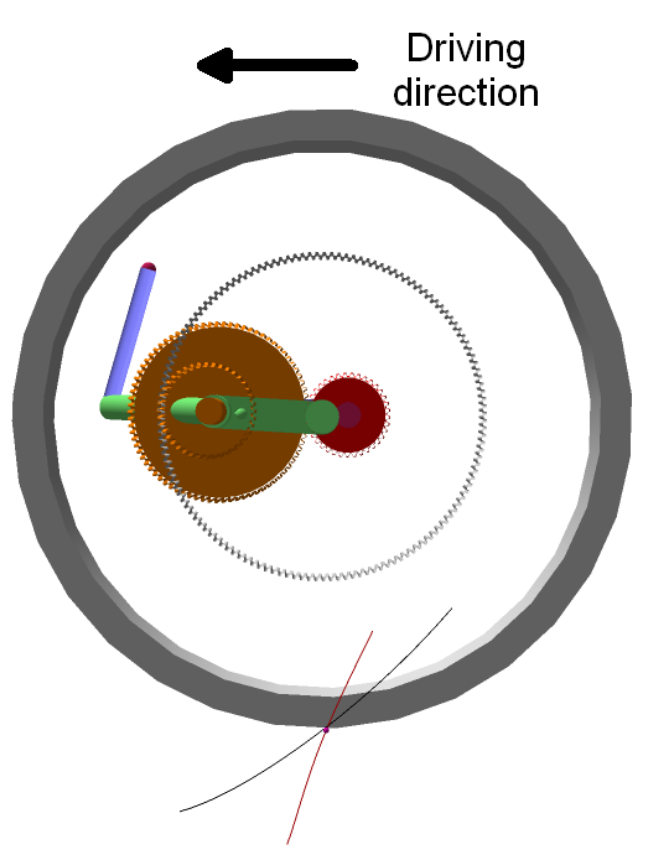

Figure 6: Trajectory of virtual tyre/road contact point of developed suspension (suspension representation simplified): Braking via electric drive (red line at the bottom) vs. conventional brake mounted on wheel carrier (black line)

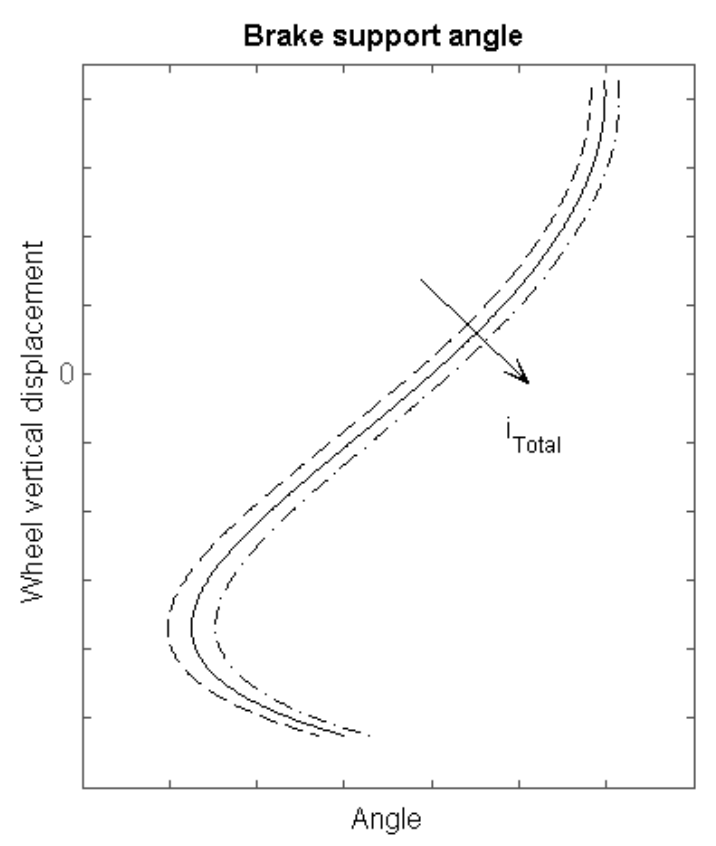

Figure 7: Brake support angle of suspension for varying gear ratio $i_{\text {Total }}$ 
discussed. Finally, some particular kinematic characteristics of the suspension are discussed in more detail.

The incorporation of the suspension into the complete vehicle model and the comparison of the simulation results with the real driving manoeuvres as well as the utilised drive control strategy will be addressed in the future.

\section{Acknowledgements}

The development of the presented suspension with integrated electric drive was partly supported by "Bayerische Forschungsstiftung" under contract AZ-840-08 for the project Fahrwerk/Antrieb Integration ins Rad (FAIR).

\section{References}

[1] Pruckner A.: Potenziale eines radnahen Elektroantriebs zur Gestaltung neuer Antriebsund Fahrzeugarchitekturen. VDI-Fachkonferenz Berechnung und Erprobung bei alternativen Antrieben. Baden-Baden, 2011

[2] Dempsey M., Gäfvert M., Harman P., Kral Ch., Otter M. and Treffinger P.: Coordinated automotive libraries for vehicle system modelling. In: Proceedings of the 5th International Modelica Conference. Vienna, 2006.

[3] Tobolář J., Otter M., Bünte T.: Modelling of Vehicle Powertrains with the Modelica PowerTrain Library. In: Proceedings of the Dynamisches Gesamtsystemverhalten von Fahrzeugantrieben. Haus der Technik Essen, Augsburg, 2007.

[4] Schweiger Ch., Dempsey M. and Otter M.: The PowerTrain Library: New Concepts and New Fields of Application. In: Proceedings of the 4th International Modelica Conference. HamburgHarburg, 2005.

[5] Pelchen Ch., Schweiger Ch., Otter M.: Modeling and Simulating the Efficiency of Gearboxes and of Planetary Gearboxes. In: Proceedings of the 2nd International Modelica Conference. Oberpfaffenhofen, 2002.

[6] Otter M., Elmqvist H., Mattsson S. E.: The New Modelica MultiBody Library. In: Proceedings of the 3rd International Modelica Conference. Linköping, 2003.
[7] Matschinsky W.: Radführungen der Straßenfahrzeuge. 3rd Edition,

ISBN 978-3-540-71196-4, 2007. 